\title{
Long non-coding RNA FEZF1-AS1 facilitates cell proliferation and migration in colorectal carcinoma
}

\author{
Na Chen ${ }^{1,2, *}$, Dan Guo ${ }^{3, *}$, Qiong Xu1,2, Minhui Yang ${ }^{1,2}$, Dan Wang ${ }^{1}$, Man Peng ${ }^{1}$, \\ Yanqing Ding ${ }^{2}$, Shuang Wang ${ }^{1,2}$, Jun Zhou ${ }^{1,2}$ \\ ${ }^{1}$ Department of Pathology, Nanfang Hospital, Southern Medical University, Guangzhou 510515, China \\ ${ }^{2}$ Department of Pathology, School of Basic Medical Sciences, Southern Medical University, Guangzhou 510515, China \\ ${ }^{3}$ Department of Pharmacy, Nanfang Hospital, Southern Medical University, Guangzhou 510515, China \\ *These authors contributed equally to this work \\ Correspondence to: Jun Zhou, e-mail: jzhou16@163.com \\ Shuang Wang, e-mail: shuangw@126.com
}

Keywords: FEZF1-AS1, FEZF1, colorectal cancer, long non-coding RNA

Received: July 16, 2015

Accepted: January 21, 2016

Published: February 03, 2016

\section{ABSTRACT}

Long non-coding RNAs (IncRNA) have been shown to play important roles in the development and progression of cancer. Here, we discovered a novel long noncoding RNA (IncRNA) FEZF1 antisense RNA1 (FEZF1-AS1) is markedly upregulated in human primary colorectal carcinoma (CRC) and associated with CRC metastasis and poor prognosis. Moreover, the downregulation of FEZF1-AS1 expression significantly inhibited the CRC cells proliferation, migration and invasiveness, suppressed S-phase entry in vitro, and repressed tumor growth and metastasis in vivo. In contrast, overexpression of FEZF1-AS1 could promote the aggressive behaviors of CRC cells. We further discovered that the downregulation of FEZF1-AS1 reduced its sense-cognate gene FEZF1 mRNA and protein expression in CRC cells. There was a positive correlation between FEZF1-AS1 and FEZF1 expression in CRC. Moreover, FEZF1 knockdown also significantly suppressed CRC cell proliferation, migration, and invasion. Our findings indicate that the dysregulation of FEZF1-AS1 participates in colorectal tumorigenesis and progression, which might be achieved, at least in part, through FEZF1 induction.

\section{INTRODUCTION}

Colorectal carcinoma (CRC) is one of the most common cancers in the world. In China, CRC ranks fifth among cancer deaths and its incidence is increasing continuously [1]. Though alterations in oncogenes and tumour suppressor genes have been reported in CRC [2], the precise molecular mechanisms underlying CRC pathogenesis remain to be fully elucidated. The long noncoding RNAs (lncRNAs) are a class of transcribed RNA molecules over 200 nucleotides with no proteincoding capacity [3]. Through regulating gene expression, lncRNAs have been shown to play crucial roles in multiple biological processes such as development, differentiation and carcinogenesis [4-7]. Dysregulated lncRNA expression has been reported in various types of cancers, such as bladder, prostate, lung, breast, gastric and colorectal cancers [8-15], indicating that lncRNAs may be involved in tumorigenesis or tumor progression.
FEZ family zinc finger 1 antisense RNA 1 (FEZF1-AS1) which located on the opposite strand of gene $F E Z F 1$, is a recently identified long non-coding RNA. However, its function in normal or cancer cells remain unknowns. In this study, we first identified that the up-regulation of IncRNA FEZF1-AS1 was associated with aggressive phenotypes of CRC and the poor prognosis in patients with CRC. Further function experiments revealed that FEZF1-AS1 increase cell proliferation, migration and invasiveness. Moreover, FEZF1-AS1 knockdown reduced its sense-cognate gene FEZF1 mRNA and protein expression in CRC cells, and there was a positive correlation between FEZF1$A S 1$ and FEZF1 expression in CRC. Furthermore, we validated that FEZF1 knockdown also significantly suppressed CRC cell proliferation, migration, and invasion. Taken together, these results suggest that FEZF1-AS1 participates as a non-coding oncogene in $\mathrm{CRC}$ carcinogenesis and metastasis. 


\section{RESULTS}

\section{LncRNA FEZF1-AS1 is up-regulated in human CRC tissues and associated with metastasis}

Using real-time RT-PCR, we detected the expression levels of FEZF1-AS1 in 34 pairs of CRC and adjacent non-neoplastic mucosa tissues. The results indicated that FEZF1-AS1 expression levels in tumor tissues of CRC patients were significantly higher than those in corresponding normal tissues ( $p=0.004$, Figure 1A). It was observed that the up-regulation of FEZF1-AS1 in tumor samples was associated with lymph-node metastasis to a significant extent ( $p=0.041$, Figure 1B). In patients diagnosed with lymph node metastases, the relative mean expression of FEZF1-AS1 was over 2.30 fold higher than that in patients without metastases $(0.00076 \pm 0.00020$ $v s .0 .00033 \pm 0.00006)$. This association indicated that FEZF1-AS1 might have a pivotal role in CRC metastasis.

\section{Up-regulation of $F E Z F 1-A S 1$ is associated with aggressive phenotypes of patients with $\mathrm{CRC}$}

To explore whether FEZF1-AS1 expression levels are associated with the clinicopathological factors of CRC, we measured FEZF1-AS1 expression in a large cohort of 153 archived paraffin-embedded CRC and normal colon tissues using in situ hybridization. We found FEZF1$A S 1$-specific staining was observed in the cytoplasm of benign and malignant epithelial cells (Figure 1C). We also observed that $89 / 153(58.17 \%)$ CRCs had highlevel expression of FEZF1-AS1, whereas 22/56 (39.29\%) normal mucosa tissues had high-level expression of FEZF1-AS1 $(p=0.015)$. The correlation analysis between clinicopathological characteristics and FEZF1-AS1 level indicated that high-level expression of FEZF1-AS1 was significantly associated with T-stage $(p=0.005)$, lymph node metastasis $(p=0.004)$, and distant metastasis $(p=0.020)$ in patients with CRC. However, it was not associated with other clinical pathological features, including patients' age, sex, tumor site, tumor size, and tumor differentiation degree $(p>0.05$, Table 1$)$.

\section{Up-regulation of $F E Z F 1-A S 1$ is correlated with poor prognosis of patients with $\mathrm{CRC}$}

To further evaluate the prognostic value of FEZF1$A S 1$ in CRCs, we analyzed the association between FEZF1-AS1 expression and survival duration using Kaplan-Meier analysis with the log-rank test. The results revealed that high-level expression of FEZF1-AS1 in CRC was significantly correlated with overall survival (Log Rank $=9.333, p=0.002$, Figure 1D) and disease-free survival $(\log$ Rank $=9.329, p=0.002$, Figure 1E) of CRC patients. The high-level of FEZF1-AS1 was associated with short survival time $(53.53 \pm 3.86 v s .79 .40 \pm 4.02)$.
To determine whether the expression of FEZF1-ASI was an independent prognostic factor for CRC, univariate and multivariate analyses were performed. The results indicated that high-level expression of FEZF1-AS1 was considered as an independent prognostic factor of outcomes in patients with CRC ( $p=0.035$, Table 2$)$.

\section{FEZF1-AS1 promotes CRC cell proliferation and colony formation in vitro}

We measured the expression level of FEZF1-AS1 in a panel of CRC cell lines (Figure 2A), and found that there were higher expression level of FEZF1-AS1 in HCT116, M5 and LOVO cell line. So, we firstly inhibited the endogenous expression of FEZF1-AS1 in M5 and HCT116 cells by shRNA to further investigate the biological effects of FEZF1-AS1 on CRCs. Moreover, FEZF1-AS1 was overexpressed in SW480 and DLD-1 cells using transfection of pcDNA-FEZF1-AS1 $(p<0.001$, Figure 2B and 2C). The growth curves determined by CCK- 8 assays indicated that cell proliferation was dramatically reduced by knockdown of endogenous FEZF1-AS1 expression in M5 and HCT116 cells, whereas overexpression of FEZF1-ASI enhanced the proliferative capacity of SW480 and DLD-1 cells $(p<0.001$, Figure 2D and 2E).

Further colony formation assays also showed that down-regulation FEZF1-AS1 expression could significantly inhibit the colony formation M5 and HCT116 cells. In contrast, overexpression of FEZF1-AS1 had the opposite effect $(p<0.05$, Figure $2 \mathrm{~F}$ and $2 \mathrm{G})$. Thus, these data revealed that FEZF1-AS1 enhances CRC cell growth.

\section{Knockdown of $F E Z F 1-A S 1$ promotes G1 arrest, but does not cause apoptosis in CRC cells}

To probe potential mechanisms by which FEZF1$A S 1$ enhanced CRC cell proliferation, we assessed cell cycle and apoptosis in CRC cell lines after FEZF1-AS1 knockdown or overexpression. Flow cytometric cell cycle assays in M5 and HCT116 cells demonstrated that FEZF1-AS1 knockdown led to a significant accumulation of cells at G0/G1-phase and a significant decrease in cells in S-phase $(p<0.05$, Figure $2 \mathrm{H})$. Conversely, the overexpressed FEZF1-AS1 mainly led to a reduction in the $\mathrm{G} 0 / \mathrm{G} 1$ population and an increase in the S-phase $(p<0.05$; Figure 2I). However, the proportion of apoptotic cells remained similar (data not shown). Thus, FEZF1-AS1induced promotion of CRC cells proliferation appeared to be mediated by modulation of the G1-S checkpoint, rather than by apoptosis.

\section{FEZF1-AS1 promotes CRC cells migration and invasion in vitro}

We also examined the effect of FEZF1-AS1 knockdown on CRC cells migration/invasion. Our 
Table 1: Correlation between the clinicopathological features and expression of FEZF1-AS1

\begin{tabular}{|c|c|c|c|c|}
\hline \multirow[b]{2}{*}{ Characteristics } & \multirow[b]{2}{*}{$n$} & \multicolumn{3}{|c|}{ FEZF1-AS1 expression } \\
\hline & & low $(\%)$ & high (\%) & $P$ value \\
\hline \multicolumn{5}{|l|}{ Gender } \\
\hline Male & 95 & $35(36.8)$ & $60(63.2)$ & \\
\hline Female & 58 & $29(50.0)$ & $29(50.0)$ & 0.109 \\
\hline \multicolumn{5}{|l|}{ Age (years) } \\
\hline$<50$ & 73 & $32(49.3)$ & $37(50.7)$ & \\
\hline$\geq 50$ & 80 & $28(35.0)$ & $52(65.0)$ & 0.073 \\
\hline \multicolumn{5}{|l|}{ Tumor site } \\
\hline Proximal colon & 47 & $22(46.8)$ & $25(53.2)$ & \\
\hline Distal colon & 37 & $16(43.2)$ & $21(56.8)$ & \\
\hline Rectum & 69 & $26(37.7)$ & $437(62.3)$ & 0.607 \\
\hline \multicolumn{5}{|c|}{ Tumor size (cm in diameter) } \\
\hline$<5$ & 50 & $17(34.0)$ & $33(66.0)$ & \\
\hline$\geq 5$ & 103 & $47(45.6)$ & $56(54.4)$ & 0.171 \\
\hline \multicolumn{5}{|l|}{ Tumor differentiation } \\
\hline Good & 15 & $8(53.3)$ & $7(46.7)$ & \\
\hline Moderate & 109 & $40(36.7)$ & $69(63.3)$ & \\
\hline Poor & 29 & $16(55.2)$ & $13(44.8)$ & 0.128 \\
\hline \multicolumn{5}{|l|}{ T-stage } \\
\hline $1-2$ & 22 & $16(72.7)$ & $6(27.3)$ & \\
\hline 3 & 122 & $47(38.5)$ & $75(61.5)$ & \\
\hline 4 & 9 & $2(22.2)$ & $7(77.8)$ & 0.005 \\
\hline \multicolumn{5}{|l|}{ N-stage } \\
\hline 0 & 92 & $47(51.1)$ & $45(48.9)$ & \\
\hline $1-2$ & 61 & $17(27.9)$ & $44(72.1)$ & 0.004 \\
\hline \multicolumn{5}{|l|}{ M-stage } \\
\hline 0 & 109 & $52(47.7)$ & $57(52.3)$ & \\
\hline 1 & 44 & $12(27.2)$ & $32(72.8)$ & 0.020 \\
\hline
\end{tabular}

results showed that the FEZF1-AS1 knockdown inhibited cell invasion by $93.0 \%$ in M5 cells, and by $88.6 \%$ in HCT116, respectively ( $p<0.001$, Figure 3A) using Matrigel invasion assay. The wound-healing assay also illustrated that FEZF1-AS1 knockdown in CRC cells caused a significant decrease in cell migration $(p<0.001$, Figure $3 \mathrm{~B})$. In contrast, invasion and migration were increased by FEZF1-AS1 overexpression in DLD-1 and SW480 cells ( $p<0.001$, Figure $3 \mathrm{C}$ and $3 \mathrm{D})$ ). These results indicated that FEZF1-AS1 facilitated CRC cell migration and invasion in vitro.

\section{Knockdown of FEZF1-AS1 inhibits tumor growth and metastasis in nude mice}

The effect of FEZF1-AS1 on tumor growth was assessed in vivo by administering a subcutaneous injection of HCT116 with stable knockdown of FEZF1-AS1 and mock cells into the subcutaneous bilateral hind leg of mice. During a 30-day follow up period, the average tumor volume in HCT116 cells with FEZF1-AS1 knockdown was reduced compared with mock cells. Moreover, using immunohistochemical staining of resected tumor tissues for $\mathrm{Ki}-67$, we found that the proliferation index was reduced in tumor tissues formed from FEZF1-AS1 knockdown, compared with those formed from control cells ( $p<0.05$, Figure 4A-4C).

The effect on metastasis was evaluated 8 weeks after the mice had been injected with the same cells through tail vein. We examined the number and size of tumor metastatic nodules under a microscope in the lung. As shown in Figure 4D and 4E, the number of pulmonary metastatic nodules was clearly decreased in the knockdown of FEZF1-AS1 group compared with that of in control group $(p=0.036$,). These results indicate that FEZF1-AS1 downregulation could inhibit tumor growth and metastasis in vivo. 


\section{Konckdown of FEZF1-AS1 decreases the expression of $F E Z F 1 \mathrm{mRNA}$ and protein}

FEZF1-AS1 is a conserved $~ 2.6-\mathrm{kb}$ RNA transcribed from the plus strand of chromosome 7 , on the opposite strand of the gene coding FEZF1 protein (7q31.32), including 611 nucleotides of full complementarity between the first FEZF1-AS1 exon and FEZF1 exons 1 (Figure 5A). To determine the relationship between FEZF1-ASI and $F E Z F 1$, we examined the expression levels between FEZF1$A S 1$ and its sense-cognate gene $F E Z F 1$. After knockdown endogenous FEZF1-AS1 expression in M5 and HCT116 cells, the expression of FEZF1 mRNA was reduced in both two cell lines. This was confirmed in protein level by western
A

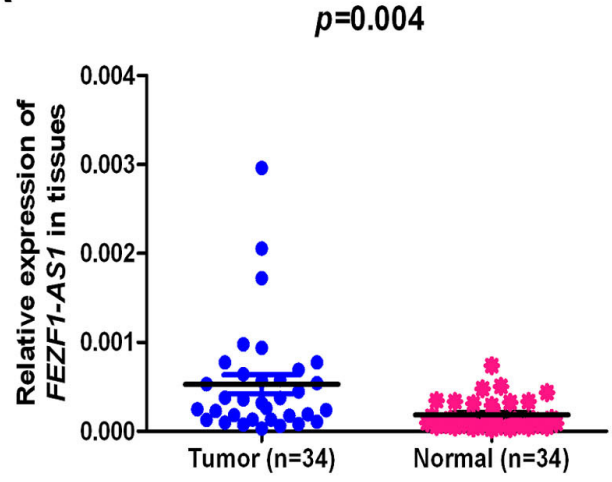

B

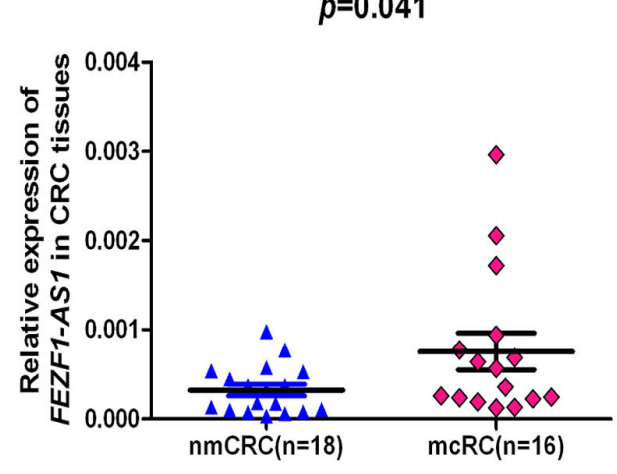

C

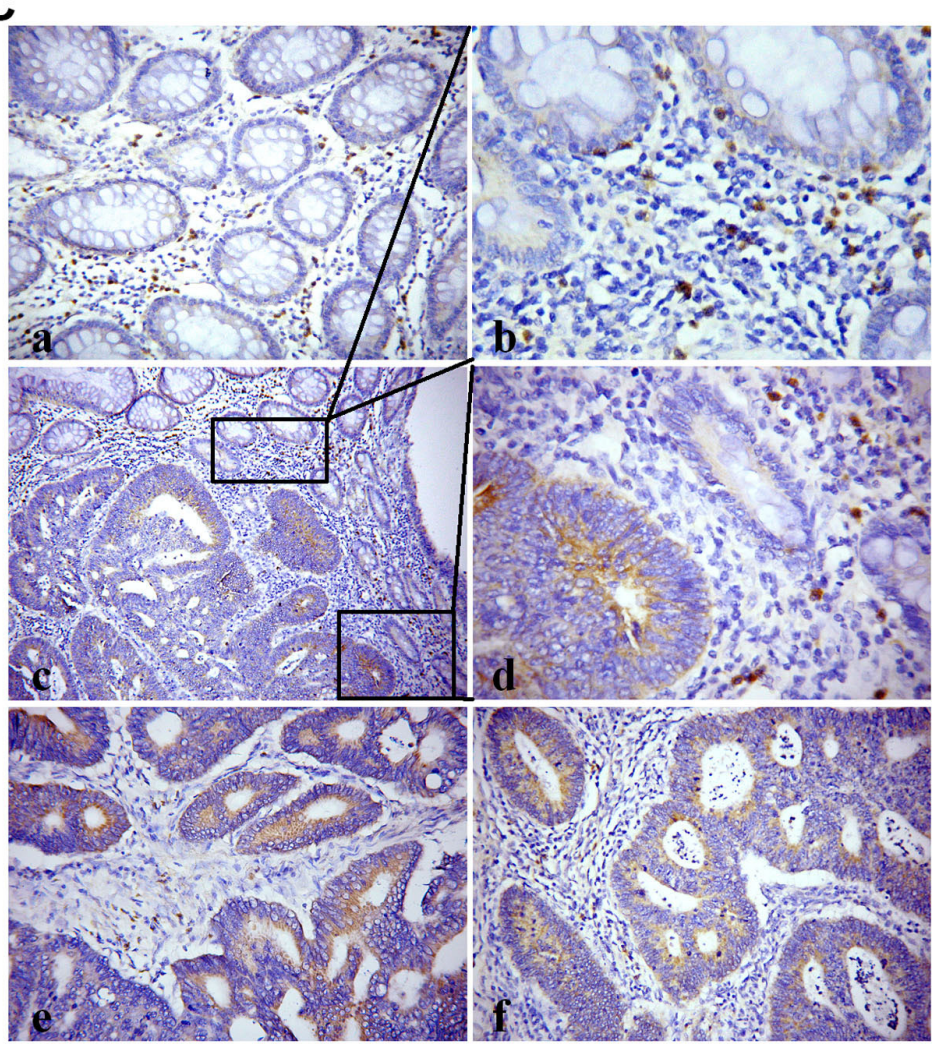

D
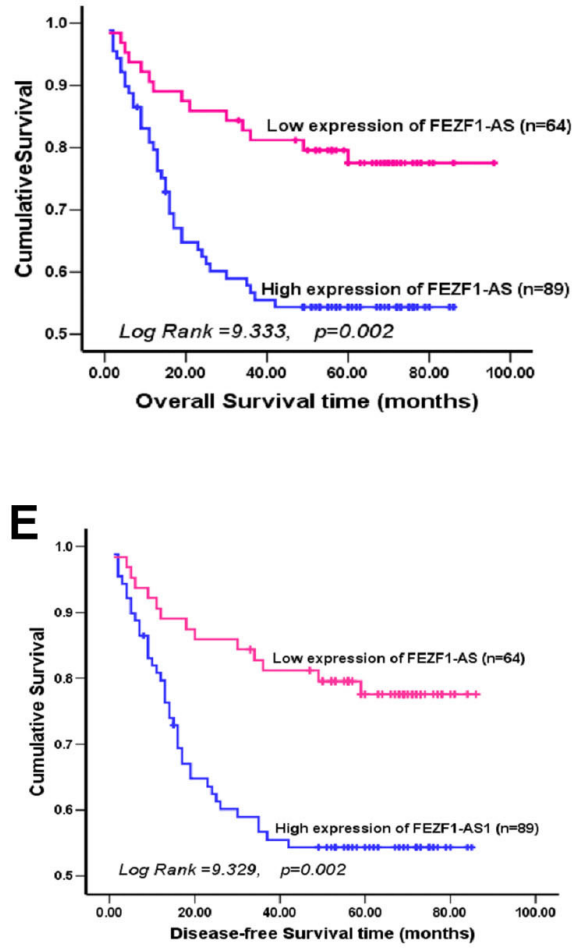

Figure 1: The levels of FEZF1-AS1 expression in CRC tissues by qRT-PCR or in situ hybridization and its prognostic value in patients with CRC. (A) Expression levels of $F E Z F 1-A S 1$ in paired CRC and adjacent normal tissues. (B) The FEZF1-ASI expression in CRC tissues with or without metastases. nmCRC denotes CRC tissues without metastases; $\mathrm{mCRC}$ denotes CRC tissues with lymph node metastases. (C) Expression analysis of FEZF1-ASI in normal colorectal mucosa and CRC tissues by in situ hybridization. (a) Negative expression of FEZF1-AS1 in normal colorectal mucosa; (b-d) High expression of FEZF1-AS1 in a tumor tissue sample and low or negative expression of FEZF1-AS1 in its normal mucosal counterpart from the same patient were observed in one filed or two independent magnification fields. (e, f) High expression of FEZF1-ASI in CRC tissues; (D, E) Kaplan-Meier analysis of (D) overall and (E) disease-free survival in all patients with CRC according to FEZF1-AS1 expression. 
Table 2: Summary of overall survival analyses by univariate and multivariate COX regression analysis

\begin{tabular}{|c|c|c|c|c|c|c|}
\hline \multirow[b]{2}{*}{ Variables } & \multicolumn{3}{|c|}{ Univariate analysis } & \multicolumn{3}{|c|}{ Multivariate analysis } \\
\hline & $P$ value & HR & CI $(95 \%)$ & $P$ value & HR & CI (95\%) \\
\hline Gender & 0.587 & 0.856 & $0.490-1.497$ & & & \\
\hline Age & 0.576 & 0.859 & $0.504-1.464$ & & & \\
\hline Tumor site & 0.834 & 0.967 & $0.707-1.322$ & & & \\
\hline Tumor size & 0.361 & 1.32 & $0.727-2.394$ & & & \\
\hline Tumor differentiation & 0.032 & 1.739 & $1.048-2.885$ & & & \\
\hline T-stage & $<0.001$ & 1.795 & $1.318-2.444$ & 0.955 & 0.986 & $0.604-1.609$ \\
\hline $\mathrm{N}$-stage & $<0.001$ & 2.887 & $1.674-4.979$ & 0.66 & 0.825 & $0.351-1.943$ \\
\hline M-stage & $<0.001$ & 9.458 & $5.294-16.898$ & 0.137 & 2.141 & $0.786-5.836$ \\
\hline FEZF1-AS1 expression & 0.003 & 2.495 & $1.356-4.590$ & 0.035 & 2.401 & $1.065-5.411$ \\
\hline
\end{tabular}

blotting (Figure 5B and 5D). However, down-regulated of FEZF1 expression did not significantly affect FEZF1-AS1 expression in CRC cells (Figure 5C). Furthermore, we also detected the expression levels of FEZF1 in the same cohort of 34 paired tissue samples using real-time PCR, the results indicated that FEZF1 expression levels in tumor tissues of CRC patients were significantly higher than those in corresponding normal tissues ( $p<0.05$, Figure 5E). At the same time, we observed a good positive correlation between FEZF1-AS1 and FEZF1 mRNA expression (Spearman's correlation analysis, $r^{2}=0.1346 ; p=0.033$, Figure 5F). These results suggested that the expression of FEZF1 might be regulated by FEZF1-AS1.

\section{siRNA-mediated knockdown of FEZF1 inhibited CRC cell proliferation, migration and invasion in vitro}

We then tested whether FEZF1 was functionally involved in CRC tumorigenesis. siRNA transfectionmediated FEZF1 knockdown decreased cell growth relative to negative control at day 5 in both cell lines $(p<0.05$; Figure $6 \mathrm{~A}$ and $6 \mathrm{~B}$ ). Furthermore, we also examined the effect of FEZF1 knockdown on M5 cells migration/ invasion using the wound-healing and matrigel invasion assay. The results indicated that FEZF1 knockdown in M5 cells caused a significant decrease in cell migration $(p=0.009$ and 0.007 , Figure 6C). Matrigel invasion assay also showed that the FEZF1 knockdown inhibited cell invasion by $59.8 \%$ and $54.9 \%$ in M5 cells ( $p=0.002$ and 0.003 , Figure $6 \mathrm{D}$ ). These results indicated that the downregulation of FEZF1 expression was sufficient in reducing both cell migration and invasion in vitro.

\section{DISCUSSION}

It has now become widely accepted that mammalian genomes encode numerous lncRNAs [16]. Dysregulation of some lncRNAs has been shown in various types of cancers, including CRC $[5,8,11,17-22]$. However, the functions and the mechanisms behind lncRNAs in CRC remain obscure. We compared the different expression profiles of lncRNA between CRC and normal mucosa tissues and mined numerous lncRNAs with deregulation expression in CRC tissues (data not shown). Among those different expression IncRNAs, we chose and focused on IncRNA FEZF1-AS1, a previously unstudied lncRNA. To the best of our knowledge, we present the first study that evaluates the prognostic impact of FEZF1-AS1 expression and comprehensive function studies of FEZF1-AS1 in CRC. In this study, we found that the expression of FEZF1-AS1 increased in CRC tissues, and CRC patients with higher FEZF1-AS1 expression tend to have advanced T-stage, lymph node, or distant metastasis. Furthermore, a KaplanMeier analysis revealed that FEZF1-AS1 over-expression in tumor cells had a significantly worse prognostic impact on the overall survival of CRC patients. The results indicated that FEZF1-AS1 could serve as a predictor for advanced $\mathrm{CRC}$ and poor prognosis for CRC patients.

In order to highlight the function of up-regulation of FEZF1-AS1 in CRC, we further explored the critical role of FEZF1-AS1 in the progression of CRC by lossof-function and gain-of-function experiments. Our results revealed that the reducing of FEZF1-ASI expression could significantly inhibit CRC cells proliferation, migration and invasiveness, and suppress S-phase entry in vitro. In contrast, overexpression of FEZF1-AS1 could promote the aggressive behaviors of CRC cells. Furthermore, the downregulation of FEZF1-AS1 could inhibit the tumorigenesis and lung metastasis in murine model. Taken together, these findings suggested that FEZF1-AS1 might serve as an oncogene and play a promotion role in CRC development and progression. Many other lncRNAs have also been implicated in the development of multiple tumors and identified as cancer biomarkers. Examples include promotion of oesophageal adenocarcinoma cells invasion and metastasis by HNF1A$A S 1$, control of colorectal cancer cells apoptosis by ZFAS1, 
A

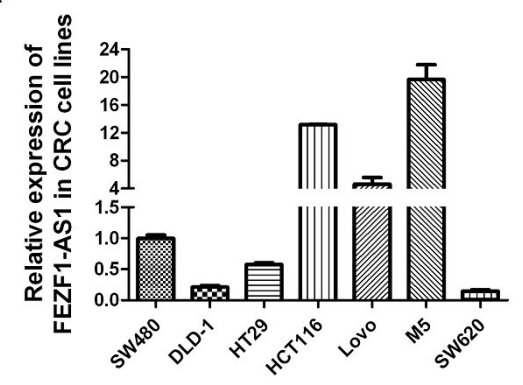

D

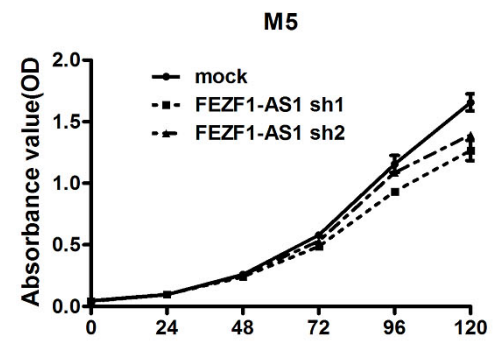

F

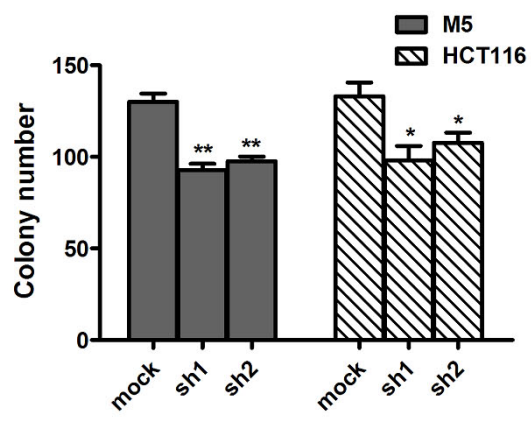

G

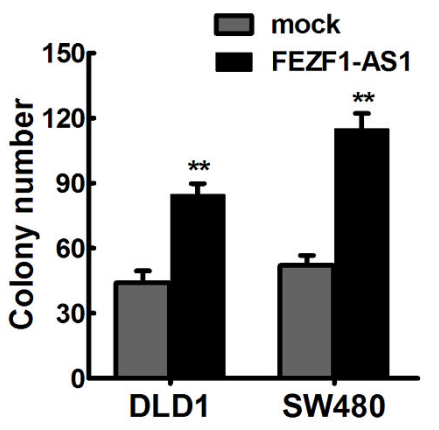

B

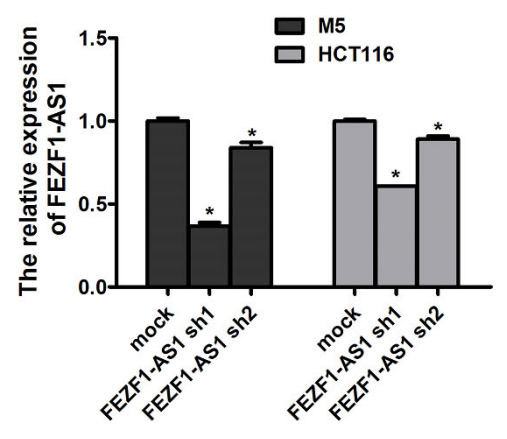

HCT 116

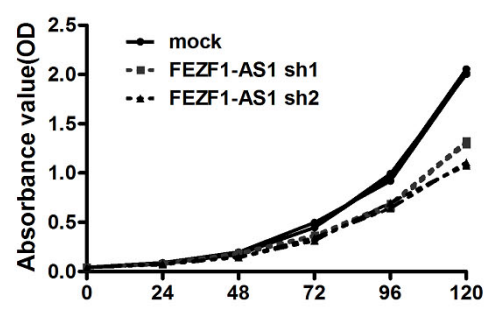

H

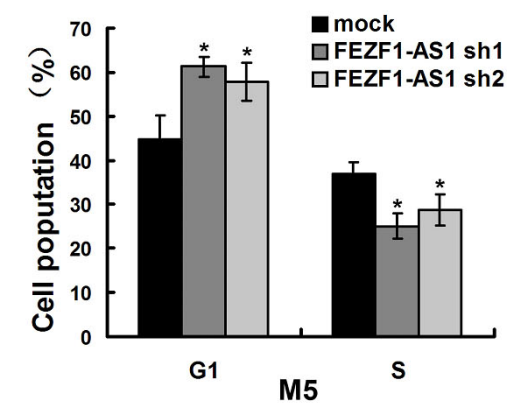

I

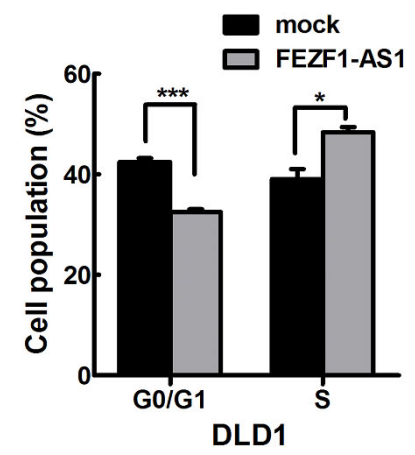

C

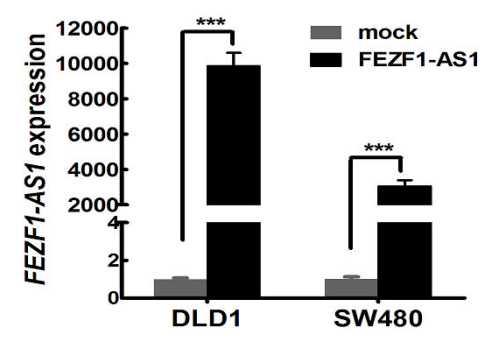

E

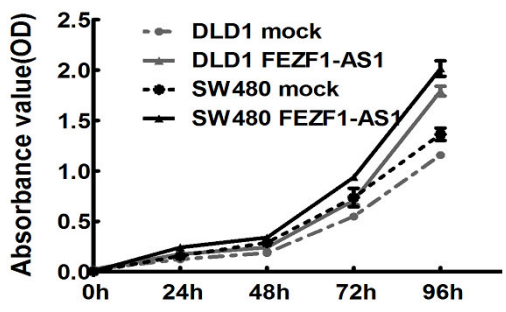

Figure 2: FEZF1-AS1 promotes CRC cell proliferation in vitro. (A) Expression of $F E Z F 1-A S 1$ in CRC cell lines and subclones by Real-time RT-PCR. (B, C) Detection of FEZF1-AS1 expression level in CRC cells after shRNA-mediated knockdown of FEZF1-ASI or overexpression of $F E Z F 1-A S 1$ by qRT-PCR. (D, E) The effect of $F E Z F 1-A S 1$ on cell proliferation was evaluated by CCK-8 assay after knockdown or overexpression of FEZF1-ASI in CRC cells. (F, G) Colony-forming assays were performed to determine the growth of CRC cells. (H, I) Cell cycle was analyzed by flow cytometry. Data were presented as mean $\pm \mathrm{SD}$. The results were reproducible in three independent experiments. ${ }^{*} p<0.05,{ }^{* *} p<0.001,{ }^{* * *} p<0.0001$. 

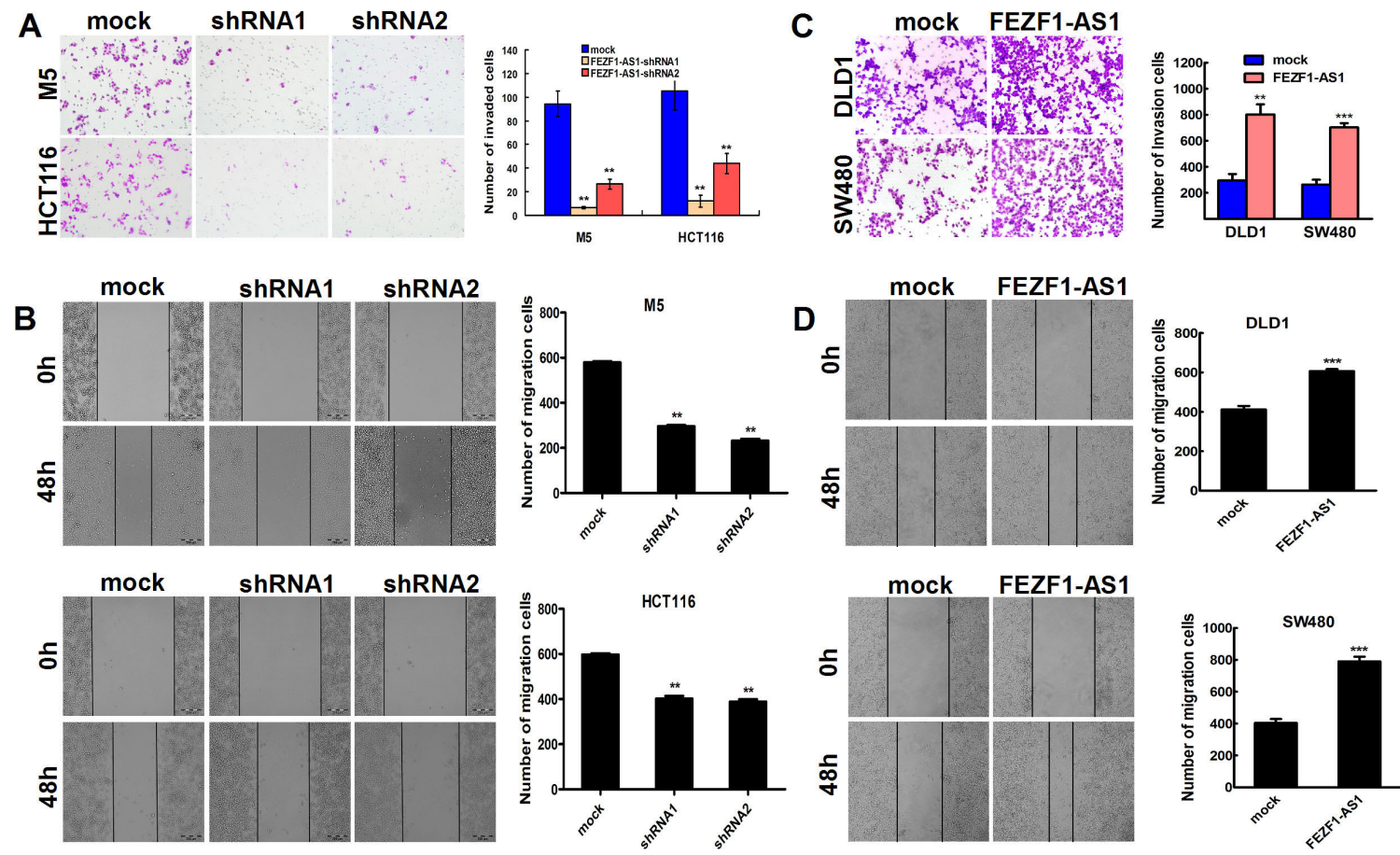

Figure 3: FEZF1-AS1 promotes migration and invasion of CRC cells in vitro. (A) Effects of decreased $F E Z F 1-A S 1$ expression on the migration potency of M5 and HCT116 were determined using matrigel invasion assay. (B) Effects of decreased FEZF1-AS1 expression on the invasion potency of in M5 and HCT116 cells were determined using scratch-wound healing assay. (C, D) Effects of overexpression FEZF1-AS1 on migration and invasion of SW480 and DLD-1 cells were determined using matrigel invasion and scratch-wound healing assay. The results were reproducible in three independent experiments. Data were presented as mean $\pm \mathrm{SD} . * * p<0.001, * * * p<0.0001$.

A

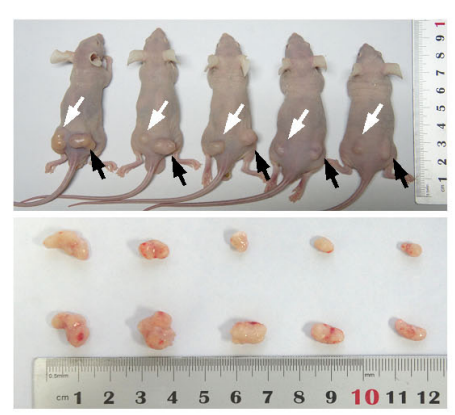

B

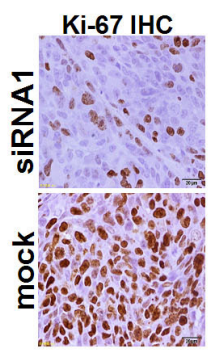

C

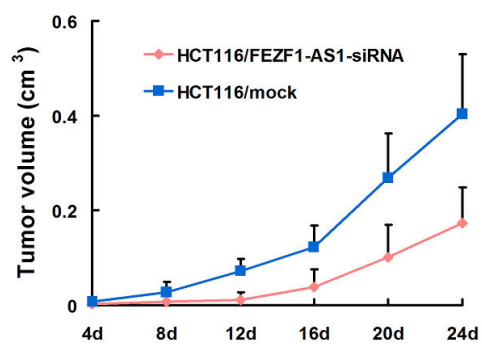

D

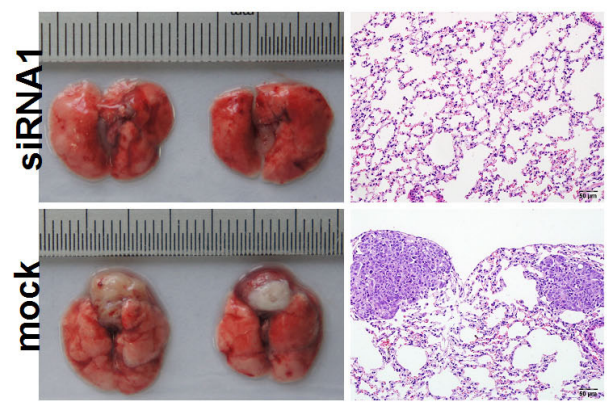

$\mathbf{E}$

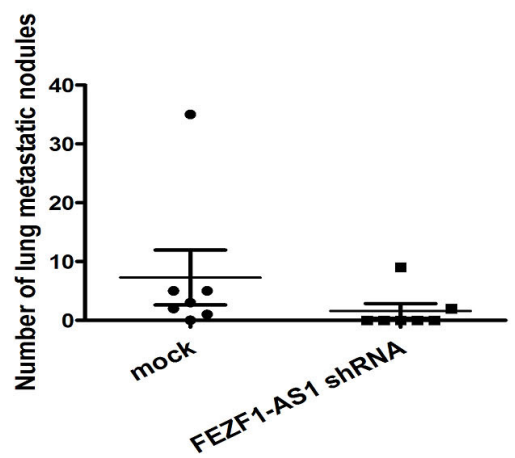

Figure 4: Knockdwon of FEZF1-AS1 repressed CRC growth, invasion and metastasis in vivo. (A) FEZF1-AS1 knockdown HCT116 and mock cells injected subcutaneously into nude mice. At 24 days after subcutaneous injection, HCT116/FEZF1-AS1-siRNA1 and HCT116/mock cells produced primary tumors (upper) and representative figure of tumor formed (lower). (B) Immunohistochemistry showed FEZF1-AS1 knockdown decreased the proliferation index Ki67 $(\times 200)$. (C) Tumor growth curve of tumor volumes. Each data point represents the mean \pm SD. (D) The whole-body images (left) and histological images (right) of metastatic nodules in lungs. (E) Lung metastatic nodules in individual mice were counted under the microscope. 
reprogramming of induced pluripotent stem cells by $R N A$ $R o R$, and regulation of non-small cell lung cancer cells growth and apoptosis by MEG3 [22-25]. Thus, effective blocking of these lncRNAs in cancer could be a novel preventive and therapeutic strategy.

The importance of IncRNAs in human cancer may be associated with their abilities to impact cellular functions through various mechanisms. Previous studies have demonstrated that the mechanisms of lncRNAs are partly dependant on their genomic location [26, 27]. It is noteworthy that some lncRNAs, which are oriented in antisense direction with respect to a protein coding loci in the opposite strand, usually act as regulator of this gene [28, 29]. FEZF1-AS1 is localized in the antisense DNA strand of the FEZF1 gene, which has been identified as critical roles in gastric cancer cell proliferation and tumorigenesis by transcriptional activation of the $K$-ras gene $[30,31]$. Therefore, we hypothesized that FEZF1AS1 might regulate FEZF1 to contribute to progression of malignant diseases. Further experiments revealed the knockdown of FEZF1-AS1 reduced FEZF1 mRNA and protein expression in $\mathrm{CRC}$ cells, and that the expression levels of FEZF1 were significantly higher in tumor tissues. Consistent to these finding, there was a positive correlation between FEZF1-AS1 and FEZF1 sense expression in CRC. These data showed that FEZF1 mRNA expression is under the control of lncRNA FEZF1-AS1. Furthermore, we validated that $F E Z F 1$ knockdown also significantly suppressed CRC cell proliferation, invasion, and migration, but did not affect the expression of FEZF1$A S 1$. Our observations indicated that the promotion effects of FEZF1-AS1 on CRC might be achieved partly by activating FEZF1 expression. Because of including 611 nucleotides of full complementarity between the exon of FEZF1-AS1 and FEZF1, we used an RNase protection assay to test the possibility of RNA duplex formation to analysis the mechanism that FEZF1-AS1 regulated FEZF1 expression. However, the results did not validated that FEZF1-AS1 formed RNA duplex with FEZF1 mRNA and increased stability of FEZF1 mRNA (data not shown). So, further studies are needed to elucidate the precise mechanisms by which FEZF1-AS1 modulates its targets.

In summary, this study provides the first link between FEZF1-AS1 expression and CRC development. We proved that the FEZF1-AS1 was upregulated in CRC tissues and its increased expression might play a promotion role in CRC tumorigenesis and progression. These results indicate that FEZF1-AS1 might be a candidate prognostic biomarker and a target for new therapies of CRC.
A

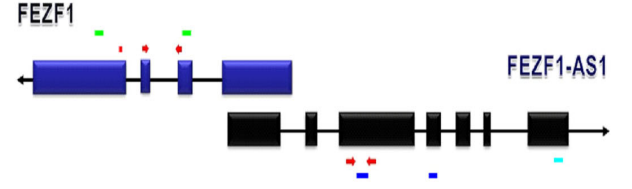

D

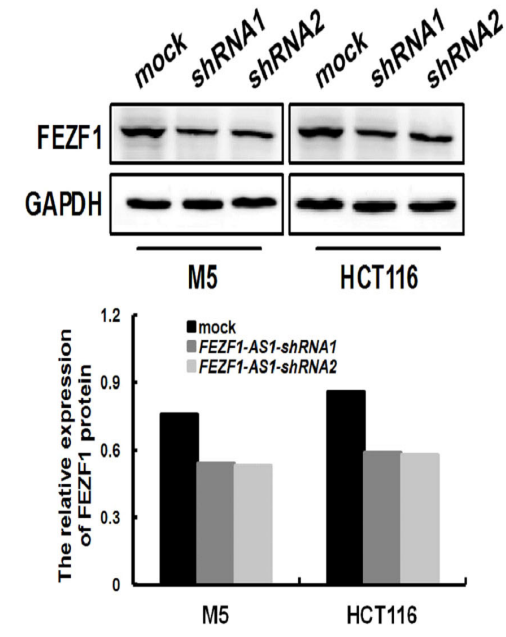

B

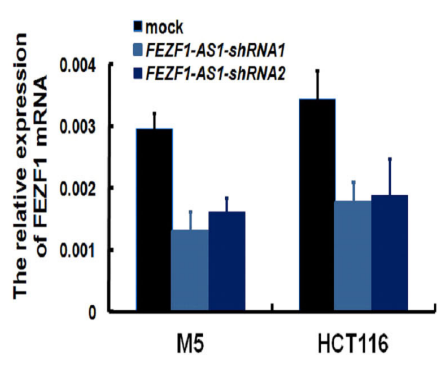

E

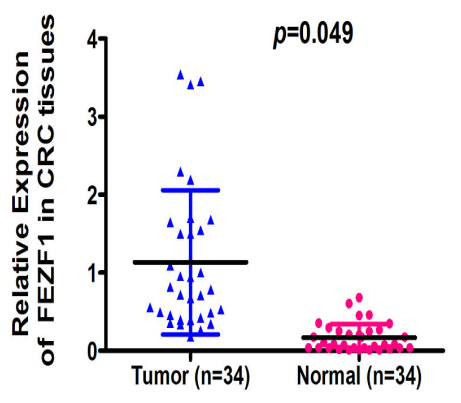

C

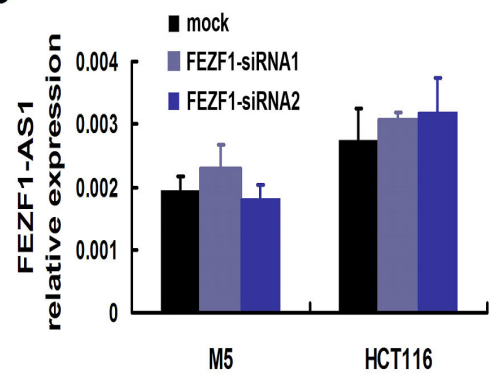

F

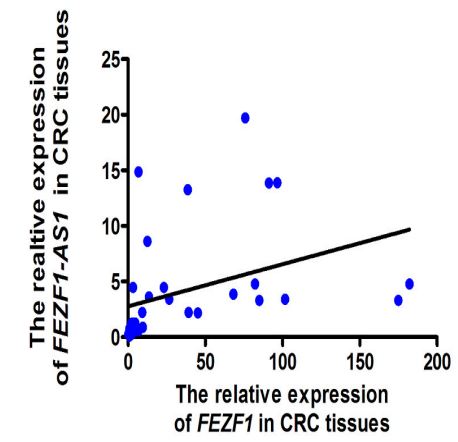

Figure 5: Konckdown of FEZF1-AS1 decreased the expression of FEZF1. (A) Schematic illustration of $F E Z F 1-A S 1$ and FEZF1 structure. There are 611 nucleotides overlapping and complementary regions between the first exon of FEZF1-AS1 and FEZF1 exon1. Red arrowhead denoted the position of PCR primers. Light green lines denoted the position of siRNA sequence of FEZF1 gene. Blue lines denoted the position of shRNA sequence of FEZF1-AS1. Light blue line denoted the location of ISH probe. (B, D) Levels of FEZF1 mRNA and protein after FEZF1-AS1-knockdown expression in CRC cell lines examined by real-time RT-PCR (B) and western blot (D). (C) Levels of FEZF1-AS1 mRNA after FEZF1 down-regulated expression in CRC cell lines examined by real-time RT-PCR. (E) Expression levels of FEZF1 in paired CRC and adjacent normal tissues by qRT-PCR. (F) Statistically significant positive correlation between FEZF1-AS1 and FEZF1 mRNA expression in CRC tissues (Spearman's correlation analysis, $r^{2}=0.1346$ ). 
A

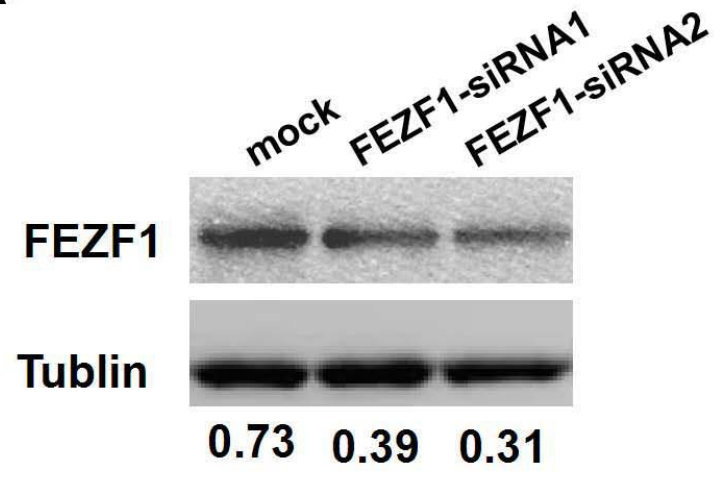

B

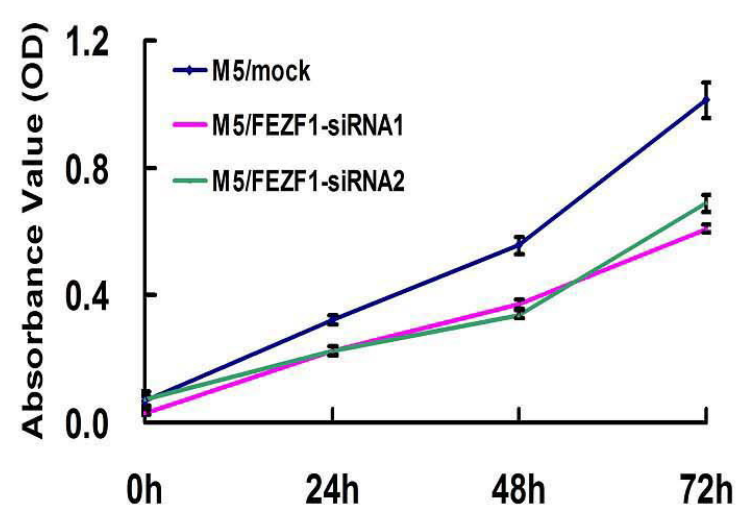

C
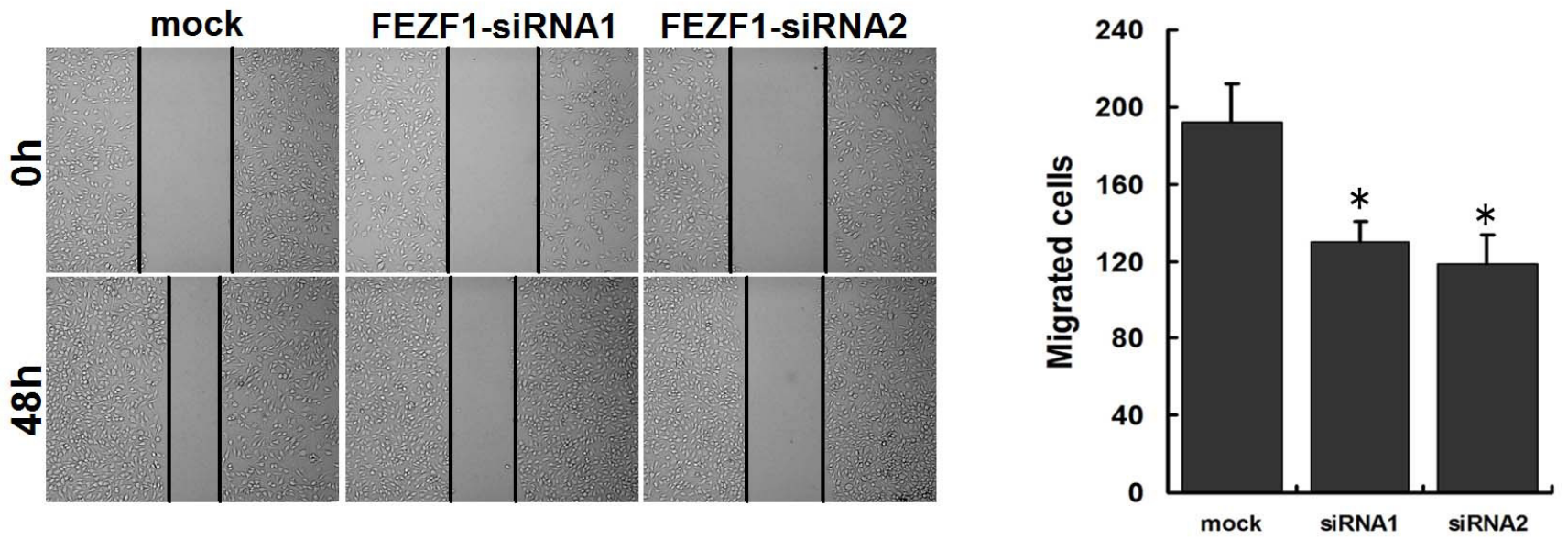

D
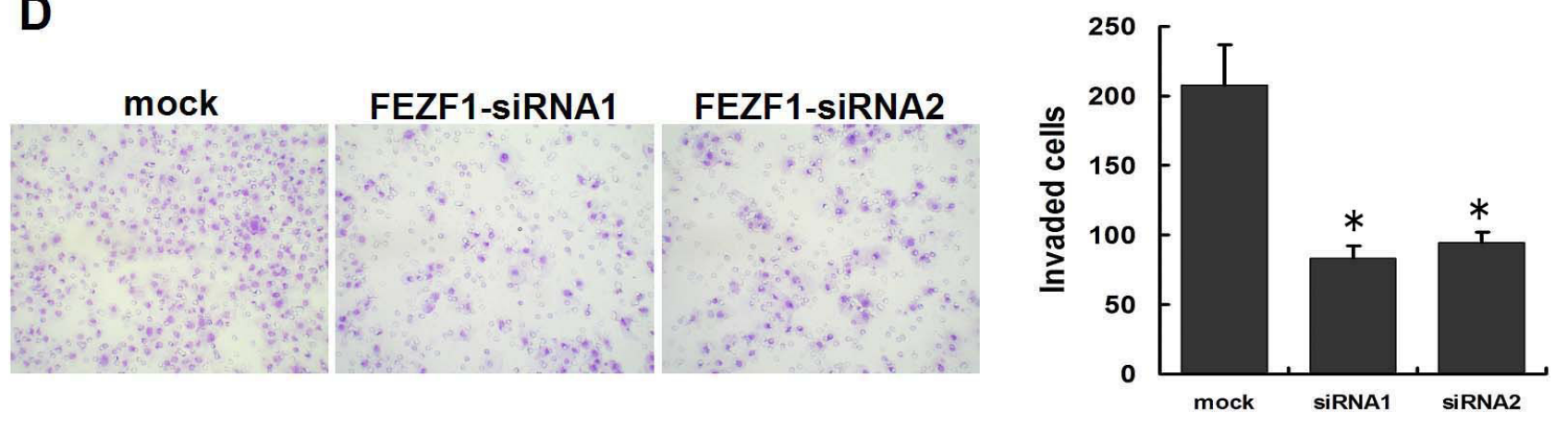

Figure 6: Knockdown of $F E Z F 1$ inhibited proliferation, invasiveness and migration of CRC cells in vitro. (A) Effects of FEZF1 siRNA on FEZF1 protein expression in M5. (B) The effect of FEZF1 on cell proliferation was evaluated by CCK-8 assay after knockdown of $F E Z F 1$ of M5. (C) Effects of decreased FEZF1 expression on the migration potency of M5 were determined using scratchwound healing assay. Data were presented as mean \pm SD. The results were reproducible in three independent experiments. (D) Effects of FEZF1-AS1 on invasiveness of M5 were determined using matrigel invasion assay. ${ }^{*} p<0.05$. 


\section{MATERIALS AND METHODS}

\section{Cell culture}

The human embryonic kidney cells 293T and the human CRC cell lines DLD-1, HT29, HCT116, SW480, SW620, Lovo were obtained from a cell bank at the Chinese Academy of Sciences (Shanghai, China). In previous studies, we described a subclone named M5 with enhanced metastatic abilities in liver [32, 33]. All CRC cell lines were cultured in RPMI 1640 medium (Gibco, Gaithersburg, MD, USA) with $10 \%$ fetal bovine serum (HyClone, Logan, USA) and $100 \mathrm{U} / \mathrm{ml}$ penicillin/ streptomycin (Gibco). They were maintained in a humidified chamber containing $5 \% \mathrm{CO}_{2}$ at $37^{\circ} \mathrm{C}$. $293 \mathrm{~T}$ was maintained in Dulbecco's modified Eagle's medium (DMEM) that was supplemented with 10\% FBS.

\section{Tissue preparation}

Fresh and formalin-fixed, paraffin-embedded, colorectal tumor tissue samples were obtained from patients who were diagnosed with primary CRC. Elective surgery was carried out on these patients at Nanfang Hospital, Southern Medical University (Guangzhou, China). In total, 34 cases of fresh CRC tissue were freshly frozen in liquid nitrogen and stored at $-80^{\circ} \mathrm{C}$ until further use. 153 cases of archived, formalin-fixed, paraffinembedded CRC tissue samples were collected and used in clinicopathological and prognostic investigation of FEZF1-AS1. A comprehensive set of clinicopathological data were recorded, including age, gender, size of primary tumor, tumor differentiation, T stage, lymph node metastasis, and distant metastasis. The stage of disease was determined according to the tumor size, lymph node, and metastasis (pTNM) classification system [34]. Complete follow-up, ranging from 1-96 months, was available for the cohort of 153 patients, and the median survival was 56 months. The use of tissues for this study has been approved by the ethics committee of Nanfang Hospital, Southern Medical University. Before using these clinical materials for research purposes, all the patients signed the informed consent. None of these patients received any preoperative chemotherapy or radiotherapy.

\section{RNA isolation and quantitative real-time PCR}

Total RNA was extracted using TRIzol Reagent (Invitrogen, Carlsbad, CA). cDNA was synthesized using the PrimeScript RT reagent Kit (Promega, Madison, WI, USA). Quantitative real-time RT-PCR was performed with the SYBR Premix EX Taq ${ }^{\text {TM }}$ (Takala, Dalian, China) using an ABI 7500 Real-Time PCR system (Applied Biosystems, Foster City, USA). GAPDH was used as an endogenous control. Fold changes were calculated through relative quantification $\left(2^{-\Delta \Delta \mathrm{Ct}}\right)$. Primers of FEZF1-AS1: 5'-TTAGGAGGCTTGTTCTGTGT-3' and
5'-GCGCAGGTACTTAAGAAAGA-3' (aligns to nucleotides $901-920$ and $1120-1139$ of the FEZF1-AS1 Reference Sequence NR_036484, respectively). GAPDH: 5'-ACAGTCAGCCGCATCTTCT-3' and 5'-GACAAGCTTCCCGTTCTCAG-3' (aligns to nucleotides 134-152 and $465-485$ of the $G A P D H$ Reference Sequence NM_001289745, respectively). FEZF1: 5'-CAGGCACAA GATCATTCACACGCAG- G-3' and 5'- CCCTTTTTGA TGAAACCCTTTGCCACAG-3' (aligns to nucleotides 986-1011 and 1118-1145 of the FEZF1 Reference Sequence NM_001024613, respectively). To account for the assessment of technical variability, the assay was performed in triplicate for each case.

\section{In situ hybridization (ISH) and evaluation of staining of FEZF1-AS1}

FEZF1-AS1 expression was examined using in situ hybridization (ISH) in CRC and non-CRC paraffinembedded sections. Briefly, after dewaxing and rehydration, the samples were digested with proteinase $\mathrm{K}$, fixed in $4 \%$ paraformaldehyde, hybridized with the 5 'digoxinlabeled probe 5'-TTCGACTGTTTCCTTGACACTAC-3' (aligns to nucleotides 2550-2572 of the FEZF1-AS1 Reference Sequence NR_036484) at $55^{\circ} \mathrm{C}$ overnight, and subsequently incubated for 30 minutes at $4^{\circ} \mathrm{C}$ with HRP. Diaminobenzidine (DAB) was used for performing color reactions. An unrelated sequence that does not match any known human gene was used as negative control.

The positive expression of FEZF1-AS1 was primarily detected in the cytoplasm. To assess the clinical characteristics of patients, the ISH stained tissue sections were reviewed and scored separately by two blinded pathologists. Based on both the intensity and proportion of FEZF1-AS1-positive cells, the staining scores were determined using a relatively simple, reproducible scoring method $[33,35]$. On a scale of 0 to 3 , the staining intensity was scored as follows: 0 (no staining), 1 (weak), 2 (medium), or 3 (strong). The extent of staining is defined as the percentage of positive staining areas of tumor cells or normal colonic epithelial cells in relation to the whole tumor area or entire section for normal samples. The extent of staining was scored on a scale of 0 to 4 as follows: 0 , $0 \% ; 1,1-25 \% ; 2,26-50 \% ; 3,51-75 \%$; and $4,76-100 \%$. The sum of the staining-intensity and staining-extent scores was used as the final staining score for FEZF1-AS1 $(0-7)$. For statistical analysis, a final staining score of $\geq 3$ was considered to be high.

\section{Vector preparation}

To reduce FEZF1-AS1 expression, two human FEZF1-AS1 shRNA sequence 5'-GCACG CTTCCGAGTTTCCATT-3' and 5'-GCCTGATGTCTA ACAGAAAGG-3' (aligns to nucleotides 1079-1099 and 1869-1889 of the FEZF1-ASI Reference Sequence NR_036484) was respectively cloned into pGU6/GFP/ 
Neo-shRNA (GenePharme, Shanghai, China). Thereafter, two FEZF1-AS1 knockdown vectors, named pGU6/ GFP/Neo-FEZF1-AS1-shRNA1 and pGU6/GFP/NeoFEZF1-AS1-shRNA2 were constructed. A scrambled shRNA oligo namely pGU6/GFP/NC-Neo-shRNA, which does not match any known human gene, was used as a control. The sequence of FEZF1-AS1 was synthesized and subcloned into pcDNA3.1 (Invitrogen, Shanghai, China). Ectopic expression of FEZF1-AS1 was achieved by using the pcDNA- $F E Z F 1-A S 1$ transfection and empty pcDNA vector (empty) was used as control. The expression level of FEZF1-AS1 was detected by qPCR.

Two human small interfering RNAs (siRNAs) targeting FEZF1 (N1, 5'-GGGTTTCTGCAGGAACT TTGA-3' and N2, 5'-GCACAAGATCATTCACACGCA-3', aligns to nucleotides 989-1009 and 1295-1315 of the FEZF1-AS1 reference sequence NR_036484, respectively) and a human scrambled siRNA sequence possessing limited homology with human genes was used as a negative control were synthesized.

\section{Lentivirus production and construction of stable cell lines}

Virus particles were harvested 48 hours after pGU6/ GFP/Neo-FEZF1-AS1-shRNA1 or -shRNA2 transfection with the packaging vector pG-P1-VSVG, pG-P2-REV and pG-P3-RRE into $293 \mathrm{~T}$ cells using lipofectamine 2000 reagent (Invitrogen). HCT116 and M5 cells were infected using the recombinant lentivirus-transducing units plus 8 $\mathrm{mg} / \mathrm{ml}$ Polybrene (Sigma, St Louis, Missouri, USA). Then, they were subjected to FACS analysis for GFP expression to gain CRC cells with stable knockdown of FEZF1-AS1.

\section{Transfection of colon cancer cells}

DLD-1, SW480, HCT116 and M5 cells were seeded into six-well plates. DLD-1 and SW480 cells were transfected with the pcDNA-FEZF-AS1 or empty vector, two FEZF1 siRNAs and control siRNA then were transfected into the HCT116 and M5 cells using Lipofectamine (Invitrogen, Carlsbad, CA) according to the manufacturer's instructions, Cells were harvested after 48 hours for qRT-PCR and western blot analyses.

\section{Cell proliferation assay}

2-(2-Methoxy-4-nitrophenyl)-3-(4-nitrophenyl)-5(2,4-disulfothenyl)-2H-tetrazolium salt (CCK-8, Dojindo, Rockville, USA) assay was conducted to evaluate the rate of cell proliferation, according to manufacturer's instructions. Briefly, log-phase cells were trypsinized into a single-cell suspension and plated into 96-well plates at a density of $2 \times 10^{3}$ per well. CCK- 8 solution was added to each well. After 1 hour, the absorbance of each well was recorded at $450 \mathrm{~nm}$ and read on a microplate reader victor (Enspire 2300 Maltilabel Reader, PerkinElmer, Singapore)

\section{Colony formation assay}

The cells were plated in 6 -well plates at $2 \times 10^{2}$ per well and maintained in RPMI1640 containing 10\% FBS for 2 weeks. After 2 weeks, the cells were washed twice with PBS, fixed with methanol and stained with $0.5 \%$ crystal violet. The number of colonies whose diameter was greater than $150 \mu \mathrm{m}$ was counted under a microscope [36].

\section{Cell migration assay}

Cell motility was measured with wound healing assay by measuring the movement of cells in a scraped, acellular area created by a $200 \mu \mathrm{L}$ pipette tube, and the spread of wound closure was observed after 0 and 48 hours, respectively. Photographs were taken to assess the level of migration in each group of transfected cells. The migration was quantified by counting the total number of cells that migrated toward the original wound field.

\section{Cell invasion assay}

For invasion assay, matrigel-coated chambers (BD Biosciences, San José, CA, USA) containing $8 \mu \mathrm{m}$ pores were used. Cells were seeded in the upper chambers (coated in matrigel) at $2 \times 10^{5}$ concentration in serum-free medium. The lower chamber of the transwell was filled with culture media containing $10 \% \mathrm{FBS}$ as a chemoattractant. After these chambers were incubated at $37^{\circ} \mathrm{C}$ for 48 hours, non-invaded cells on the top of the transwell were scraped off with a cotton swab. The successfully translocated cells were fixed with $10 \%$ formalin. Then, they were stained with $0.1 \%$ crystal violet for 30 minutes and counted under a light microscope.

\section{Flow cytometric analysis}

Cells were seeded at a density of $1 \times 10^{6}$ cells/well in six well plates. After $24 \mathrm{~h}$, cells were washed with PBS and fixed in ice-cold $70 \%$ ethanol for $1 \mathrm{~h}$ and then treated with $100 \mu \mathrm{L}$ of $50 \mathrm{mg} / \mathrm{L}$ propidium iodide for $30 \mathrm{~min}$ at $4^{\circ} \mathrm{C}$ in the dark. The cell cycle profiles were assayed using the Elite ESP flow cytometer at $488 \mathrm{~nm}$, and data were analyzed with the CELL Quest software (BD Biosciences, San Jose, CA, USA).

\section{Animal experiments}

Balb/C-nu/nu athymic nude mice (4-6 weeks old) were obtained from the Laboratory Animal Centre of Southern Medical University. For tumor growth assay, a total of $2 \times 10^{6}$ cells suspended of HCT116 with stable knockdown of FEZF1-AS1, or mock cells, were respectively injected into left and right bilateral hind leg subcutaneous of mice. The tumor size was measured using digital calipers every three days. After 24 days of monitoring, mice were sacrificed by cervical dislocation and tumors were dissected. Tumor volume was calculated 
as follows: Volume $=\left(\mathrm{D} \times \mathrm{d}^{2}\right) / 2$, where $\mathrm{D}$ meant the longest diameter and $\mathrm{d}$ meant the shortest diameter.

For the in vivo metastasis assay, the mice were i.v. injected via the lateral tail vein with $2 \times 10^{6}$ tumor cells or control cells were suspended in $0.1 \mathrm{~mL}$ of culture medium. Animal health status was monitored every three days. Human endpoints had been planned at the end of experiment as a means to relieve pain or distress. At the time of euthanasia, the lungs were removed by dissection away from adjacent organs and fixed with $10 \%$ neutral buffered formalin. Subsequently, the consecutive tissue sections were obtained and stained with haematoxylineosin (H \& E) to observe the metastatic nodules of lungs under microscope. All the experimental procedures were performed in strict accordance with the recommendations in the Guide for the Care and Use of Laboratory Animals of the National Institutes of Health. The protocol was approved by the Committee on the Ethics of Animal Experiments of Southern Medical University. All the necessary steps were taken to minimize the suffering and distress caused to the mice.

\section{Statistical analysis}

All statistical analyses were performed using the SPSS 16.0 statistical software package. In at least three independent experiments, the data were presented in terms of mean \pm SEM. The differences between variables were assessed using three statistical tests: $\chi^{2}$ test, Fisher's exact test, or One-way ANOVA. For patients with different levels of FEZF1-AS1 expression, the survival curves were plotted using the Kaplan-Meier method and compared using the log-rank test. The multivariate survival analysis was performed on all parameters that were found to be significant in univariate analysis using the Cox regression model. A $p$ value less than 0.05 was considered as statistical significance.

\section{GRANT SUPPORT}

This study was supported by National Nature Science Fund of China (No. 81272763, 81000953, 81172242 and 81472318) and the Natural Science Foundation of Guangdong Province (2014A030313286 and 2014A030313308).

\section{CONFLICTS OF INTEREST}

The authors declare no conflicts of interest.

\section{REFERENCES}

1. Sung JJ, Lau JY, Young GP, Sano Y, Chiu HM, Byeon JS, Yeoh KG, Goh KL, Sollano J, Rerknimitr R, Matsuda T, $\mathrm{Wu} \mathrm{KC}, \mathrm{Ng} \mathrm{S}$, et al. Asia Pacific consensus recommendations for colorectal cancer screening. Gut. 2008; 57:1166-1176.
2. De Rosa M, Pace U, Rega D, Costabile V, Duraturo F, Izzo P, Delrio P. Genetics, diagnosis and management of colorectal cancer (Review). Oncol Rep. 2015; 34:1087-1096.

3. Tsai MC, Spitale RC, Chang HY. Long intergenic noncoding RNAs: new links in cancer progression. Cancer Res. 2011; 71:3-7.

4. Huarte M, Rinn JL. Large non-coding RNAs: missing links in cancer? Hum Mol Genet. 2010; 19:R152-161.

5. Guttman M, Donaghey J, Carey BW, Garber M, Grenier JK, Munson G, Young G, Lucas AB, Ach R, Bruhn L, Yang X, Amit I, Meissner A, et al. lincRNAs act in the circuitry controlling pluripotency and differentiation. Nature. 2011; 477:295-300.

6. Cai B, Song XQ, Cai JP, Zhang S. HOTAIR: a cancer-related long non-coding RNA. Neoplasma. 2014; 61:379-391.

7. Gomez-Maldonado L, Tiana M, Roche O, Prado-Cabrero A, Jensen L, Fernandez-Barral A, Guijarro-Munoz I, Favaro E, Moreno-Bueno G, Sanz L, Aragones J, Harris A, Volpert O, et al. EFNA3 long noncoding RNAs induced by hypoxia promote metastatic dissemination. Oncogene. 2014; 34:2609-2620.

8. Gupta RA, Shah N, Wang KC, Kim J, Horlings HM, Wong DJ, Tsai MC, Hung T, Argani P, Rinn JL, Wang Y, Brzoska P, Kong B, et al. Long non-coding RNA HOTAIR reprograms chromatin state to promote cancer metastasis. Nature. 2010; 464:1071-1076.

9. Gutschner T, Hammerle M, Eissmann M, Hsu J, Kim Y, Hung G, Revenko A, Arun G, Stentrup M, Gross M, Zornig M, MacLeod AR, Spector DL, et al. The noncoding RNA MALAT1 is a critical regulator of the metastasis phenotype of lung cancer cells. Cancer Res. 2013; 73:1180-1189.

10. Fan Y, Shen B, Tan M, Mu X, Qin Y, Zhang F, Liu Y. TGFbeta-induced upregulation of malat1 promotes bladder cancer metastasis by associating with suz12. Clin Cancer Res. 2014; 20:1531-1541.

11. Wang J, Liu X, Wu H, Ni P, Gu Z, Qiao Y, Chen N, Sun F, Fan Q. CREB up-regulates long non-coding RNA, HULC expression through interaction with microRNA-372 in liver cancer. Nucleic Acids Res. 2010; 38:5366-5383.

12. Jin G, Sun J, Isaacs SD, Wiley KE, Kim ST, Chu LW, Zhang Z, Zhao H, Zheng SL, Isaacs WB, Xu J. Human polymorphisms at long non-coding RNAs (lncRNAs) and association with prostate cancer risk. Carcinogenesis. 2011; 32:1655-1659.

13. Ji Q, Zhang L, Liu X, Zhou L, Wang W, Han Z, Sui H, Tang Y, Wang Y, Liu N, Ren J, Hou F, Li Q. Long noncoding RNA MALAT1 promotes tumour growth and metastasis in colorectal cancer through binding to SFPQ and releasing oncogene PTBP2 from SFPQ/PTBP2 complex. Br J Cancer. 2014; 111:736-748.

14. Xie M, Sun M, Zhu YN, Xia R, Liu YW, Ding J, Ma HW, He XZ, Zhang ZH, Liu ZJ, Liu XH, De W. Long noncoding RNA HOXAAS2 promotes gastric cancer proliferation by epigenetically silencing P21/PLK3/DDIT3 expression. Oncotarget. 2015; 6:33587-33601. doi: 10.18632/oncotarget.5599. 
15. Li Y, Li Y, Chen W, He F, Tan Z, Zheng J, Wang W, Zhao Q, Li J. NEAT expression is associated with tumor recurrence and unfavorable prognosis in colorectal cancer. Oncotarget. 2015; 6:27641-27650. doi: 10.18632/oncotarget.4737.

16. Guttman M, Amit I, Garber M, French C, Lin MF, Feldser D, Huarte M, Zuk O, Carey BW, Cassady JP, Cabili MN, Jaenisch R, Mikkelsen TS, et al. Chromatin signature reveals over a thousand highly conserved large non-coding RNAs in mammals. Nature. 2009; 458:223-227.

17. Khaitan D, Dinger ME, Mazar J, Crawford J, Smith MA, Mattick JS, Perera RJ. The melanoma-upregulated long noncoding RNA SPRY4-IT1 modulates apoptosis and invasion. Cancer Res. 2011; 71:3852-3862.

18. Cui Z, Ren S, Lu J, Wang F, Xu W, Sun Y, Wei M, Chen J, Gao X, Xu C, Mao JH, Sun Y. The prostate cancer-upregulated long noncoding RNA PlncRNA-1 modulates apoptosis and proliferation through reciprocal regulation of androgen receptor. Urol Oncol. 2012; 31:1117-1123.

19. Wang Y, Chen W, Yang C, Wu W, Wu S, Qin X, Li X. Long non-coding RNA UCA1a(CUDR) promotes proliferation and tumorigenesis of bladder cancer. Int J Oncol. 2012; 41:276-284.

20. Hu Y, Chen HY, Yu CY, Xu J, Wang JL, Qian J, Zhang X, Fang JY. A long non-coding RNA signature to improve prognosis prediction of colorectal cancer. Oncotarget. 2014; 5:2230-2242. doi: 10.18632/oncotarget.1895.

21. Ma Y, Yang Y, Wang F, Moyer MP, Wei Q, Zhang P, Yang Z, Liu W, Zhang H, Chen N, Wang H, Wang H, Qin H. Long non-coding RNA CCAL regulates colorectal cancer progression by activating Wnt/beta-catenin signalling pathway via suppression of activator protein 2alpha. Gut. 2015. doi: 10.1136/gutjnl-2014-308392.

22. Yang $\mathrm{X}$, Song JH, Cheng $\mathrm{Y}, \mathrm{Wu} \mathrm{W}$, Bhagat $\mathrm{T}$, Yu Y, Abraham JM, Ibrahim S, Ravich W, Roland BC, Khashab M, Singh VK, Shin EJ, et al. Long non-coding RNA HNF1A-AS1 regulates proliferation and migration in oesophageal adenocarcinoma cells. Gut. 2015; 63:881-890.

23. Thorenoor N, Faltejskova-Vychytilova P, Hombach S, Mlcochova J, Kretz M, Svoboda M, Slaby O. Long noncoding RNA ZFAS1 interacts with CDK1 and is involved in p53-dependent cell cycle control and apoptosis in colorectal cancer. Oncotarget. 2016; 7:622-37. doi: 10.18632/ oncotarget.5807.

24. Loewer S, Cabili MN, Guttman M, Loh YH, Thomas K, Park IH, Garber M, Curran M, Onder T, Agarwal S, Manos PD, Datta S, Lander ES, et al. Large intergenic non-coding RNARoR modulates reprogramming of human induced pluripotent stem cells. Nature genetics. 2010; 42:1113-1117.

25. Braconi C, Kogure T, Valeri N, Huang N, Nuovo G, Costinean S, Negrini M, Miotto E, Croce CM, Patel T. microRNA-29 can regulate expression of the long noncoding RNA gene MEG3 in hepatocellular cancer. Oncogene. 2011; 30:4750-4756.
26. Tian D, Sun S, Lee JT. The long noncoding RNA, Jpx, is a molecular switch for X chromosome inactivation. Cell. 2010; 143:390-403.

27. Orom UA, Derrien T, Beringer M, Gumireddy K, Gardini A, Bussotti G, Lai F, Zytnicki M, Notredame C, Huang Q, Guigo R, Shiekhattar R. Long noncoding RNAs with enhancer-like function in human cells. Cell. 2010; 143:46-58.

28. Nolasco S, Bellido J, Goncalves J, Tavares A, Zabala JC, Soares H. The expression of tubulin cofactor A (TBCA) is regulated by a noncoding antisense Tbca RNA during testis maturation. PLoS One. 2012; 7:e42536.

29. Katayama S, Tomaru Y, Kasukawa T, Waki K, Nakanishi M, Nakamura M, Nishida H, Yap CC, Suzuki M, Kawai J, Suzuki H, Carninci P, Hayashizaki Y, et al. Antisense transcription in the mammalian transcriptome. Science. 2005; 309:1564-1566.

30. Song IS, Ha GH, Kim JM, Jeong SY, Lee HC, Kim YS, Kim YJ, Kwon TK, Kim NS. Human ZNF312b oncogene is regulated by $\mathrm{Sp} 1$ binding to its promoter region through DNA demethylation and histone acetylation in gastric cancer. Int J Cancer. 2011; 129:2124-2133.

31. Song IS, Oh NS, Kim HT, Ha GH, Jeong SY, Kim JM, Kim DI, Yoo HS, Kim CH, Kim NS. Human ZNF312b promotes the progression of gastric cancer by transcriptional activation of the K-ras gene. Cancer Res. 2009; 69:3131-3139.

32. Zhang YF, Liu L, Ding YQ. Isolation and characterization of human colorectal cancer cell subline with unique metastatic potential in the liver [Article in Chinese]. Nan Fang Yi Ke Da Xue Xue Bao. 2007; 27:126-130.

33. Wang S, Zhou J, Wang XY, Hao JM, Chen JZ, Zhang XM, Jin H, Liu L, Zhang YF, Liu J, Ding YQ, Li JM. Downregulated expression of SATB2 is associated with metastasis and poor prognosis in colorectal cancer. J Pathol. 2009; 219:114-122.

34. Markowitz SD, Bertagnolli MM. Molecular origins of cancer: Molecular basis of colorectal cancer. N Engl J Med. 2009; 361:2449-2460.

35. Soumaoro LT, Uetake H, Higuchi T, Takagi Y, Enomoto M, Sugihara K. Cyclooxygenase-2 expression: a significant prognostic indicator for patients with colorectal cancer. Clin Cancer Res. 2004; 10:8465-8471.

36. Wu W, Bhagat TD, Yang X, Song JH, Cheng Y, Agarwal R, Abraham JM, Ibrahim S, Bartenstein M, Hussain Z, Suzuki M, Yu Y, Chen W, et al. Hypomethylation of noncoding DNA regions and overexpression of the long noncoding RNA, AFAP1-AS1, in Barrett's esophagus and esophageal adenocarcinoma. Gastroenterology. 2013; 144:956-966 e954. 